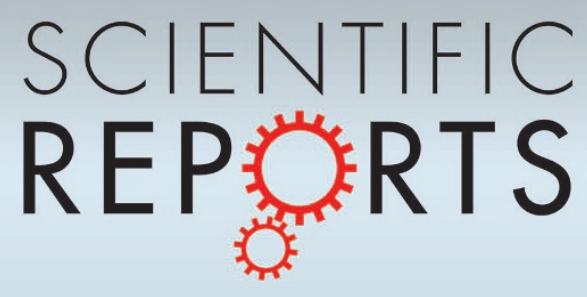

\title{
OPEN A facile method for the large-scale continuous synthesis of graphene sheets using a novel catalyst
}

SUBJECT AREAS:

MATERIALS FOR ENERGY

AND CATALYSIS

SYNTHESIS AND PROCESSING

SYNTHESIS OF GRAPHENE

ENVIRONMENTAL CHEMISTRY

Received

4 July 2013

Accepted

7 October 2013

Published

24 October 2013

Correspondence and requests for materials should be addressed to

A.C.L. (maclua@ntu. edu.sg)

\author{
Yi Shen \& Aik Chong Lua
}

School of Mechanical and Aerospace Engineering, Nanyang Technological University, 50 Nanyang Avenue, Singapore 639798, Republic of Singapore.

This study reports on a facile and economical method for the scalable continuous synthesis of graphene sheets by the thermocatalytic decomposition of methane using a unique and novel unsupported catalyst of iron particles. Single-layered and few-layered graphene sheets were continuously synthesized by the isothermal decomposition reaction of methane over a catalyst of iron particles under atmospheric pressure without the need for a cooling precipitation process. In contrast with the methods currently reported in the published literature, this method exhibits remarkably high capacity and efficiency in terms of graphene throughput and yield, respectively. A maximum graphene yield rate of $20 \mathrm{mg} / \mathrm{min}$ per $\mathrm{g}$ of catalyst and a graphene output of $6 \mathrm{~g}$ per $\mathrm{g}$ of catalyst were achieved in this study; this graphene output has far surpassed the best graphene yield of $50 \mathrm{mg}$ per $500 \mathrm{mg}$ of catalyst, thus reported so far, by 60 times.

$\longrightarrow$ raphene, a one-atom-thick sheet of carbon atoms with a two-dimensional hexagonal lattice structure, has attracted intense attention because of its unique properties such as fast charge carrier mobility, high thermal conductivity and large surface area with potential applications in energy, catalysis and sensing. The first graphene was obtained by the micromechanical cleavage of highly oriented pyrolytic graphite using adhesive Scotch tapes ${ }^{1}$. This approach had proved to be an easy production method of high quality graphene with very low concentrations of structural defects and led to many fundamental studies of its properties. However, this approach is not scalable to produce a large quantity of graphene. Subsequently, a chemical method to synthesize graphene was proposed, which involved the exfoliation and reduction of graphene oxide dispersion in inorganic solvents ${ }^{2-4}$. This method of synthesis has the advantage in producing graphene derivatives with tunable electrical and optical properties. Unfortunately, reduced graphene oxide exhibits much lower electrical conductivity when compared with the pristine graphene because of structural defects and electron disorders. The bottom-up synthesis of graphene is an alternative approach to the mechanical exfoliation of bulk graphite and the chemical reduction of exfoliated graphite oxide. In particular, chemical vapour deposition (CVD) has been widely studied to synthesize graphene on various substrate surfaces, such as $\mathrm{Ni}^{5-7}, \mathrm{Pd}^{8}, \mathrm{Ru}^{9,10}, \mathrm{Ir}^{11}, \mathrm{Cu}^{12-15}, \mathrm{Pt}^{16,17}, \mathrm{SiO}_{2}{ }^{18,19}$ and $\mathrm{SiC}^{20}$. Large-area graphene sheets were successfully synthesized by CVD processes and subsequently transferred to desired substrates but these processes are highly dependent on the surface properties of the substrates and involve laborious experimental procedures and sophisticated instrumentation. Moreover, the extremely low yield of graphene (i.e., $50 \mathrm{mg}$ of graphene sheet/500 mg of catalyst - the best yield currently reported ${ }^{21}$ ) obtained by current CVD processes is still a stumbling block to its large-scale production. Large-scale production of graphene powder is of great importance for further studies and applications of graphene ${ }^{22}$. In this study, a facile and economical method to large-scale synthesis of graphene sheets using a novel catalyst was reported. In contrast to the use of high-cost metals and ultra-high vacuum system in a previous study ${ }^{14}$, graphene sheets were continuously synthesized by the thermocatalytic decomposition of methane under atmospheric pressure using iron oxide crystals as catalyst precursors in this work.

\section{Results}

The catalyst precursors of monodispersed rod-like iron oxide particles possessed a uniform width range of 30$40 \mathrm{~nm}$ and a length ranging from 100 to $300 \mathrm{~nm}$ as shown in Fig. 1. The selected area electron diffraction (SAED) pattern (inset in Fig. 1B) indicates the single-crystalline and hexagonal close packing structure of the iron oxide. The crystallographic structure of the iron oxide was characterized by X-ray diffraction (XRD) technique. All peaks were indexed to $\alpha$ iron oxide (JCPDS: 33-0664) crystals as shown in Fig. S1 (see Supplementary information). 

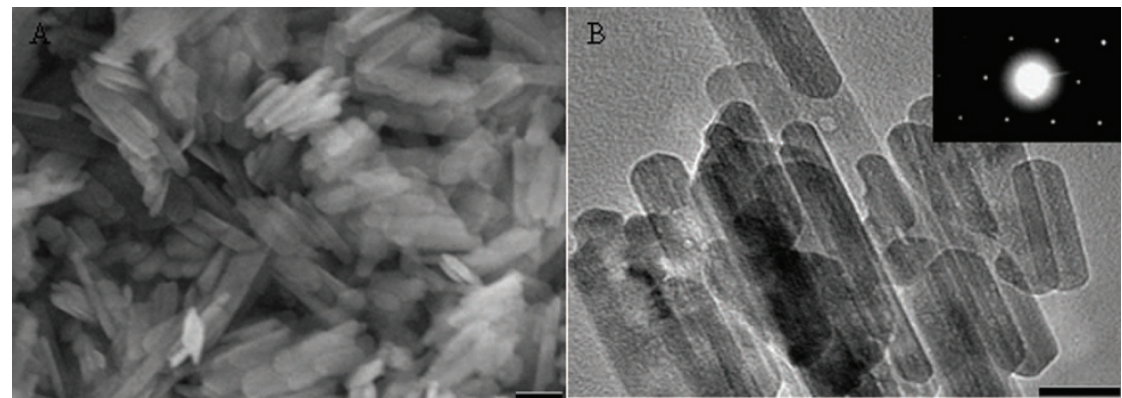

Figure 1 Electron microscopy of iron oxide particles. (A) SEM and (B) TEM micrographs of iron oxide particles (inset in (B): SAED pattern of iron oxide particle). The scale bars are (A) $100 \mathrm{~nm}$, and (B) $50 \mathrm{~nm}$.

The elemental composition of the iron oxide was examined by energy dispersive X-ray spectroscopy (EDX) as shown in Fig. S2 (see Supplementary information).

The graphene sheets produced were characterized by a number of analytical methods. Representative field emission scanning electron microscopy (FESEM) micrographs of the as-synthesized graphene sheets are shown in Figs. 2A and 2B. Due to the high efficiency of the continuous growth of graphene sheets, the iron particles were wrapped around by the graphene sheets. Wrinkles and ripples were observed on the as-synthesized graphene sheets.

Figs. $3 \mathrm{~A}$ and $3 \mathrm{~B}$ show the transmission electron microscopy (TEM) micrographs of the graphene sheets. The TEM micrograph at low magnification (Fig. 3A) shows a very distinct contrast between the graphene sheets and the catalyst particles. The black spots indicate the catalyst particles; the continuous and featureless regions are indicative of the central parts of the graphene sheets while the slightly stretched black regions are indicative of the scrolls and folds of the graphene sheets. A high-resolution TEM micrograph of a typical graphene sheet (Fig. 3B) shows the lattice fringes with a spacing of $2.13 \AA$ that corresponds to the carbon (110) plane. Fig. 3C shows the interface between the graphene sheet and the catalyst particle. The graphene sheet is closely attached to the surface of the iron particle. The spacing of the layers was measured to be about $3.4 \AA$ and it could be indexed to the (002) plane of graphite. As the graphene sheets are locally parallel to the electron beam at the folds, such folds could provide a clear TEM signature for the number of graphene layers ${ }^{23}$. The as-synthesized product consisting of graphene sheets with different layers are shown in Fig. S3 (see Supplementary information). By careful examination and analyses of a large number of TEM micrographs, the number distribution of the layers of the graphene sheets is statistically shown in Fig. 3D. The data showed that the assynthesized graphene sheets consisted of $1.7 \%$ single-layered graphene, $65 \%$ few-layered graphene sheets (2-10 layers per sheet) and $33.3 \%$ multi-layered graphene sheets (greater than 10 layers per sheet).
The crystallographic structure of the graphene sheets was characterized by SAED. Extensive SAED studies showed that most of the graphene sheets exhibited a single set of hexagonal diffraction pattern with sharp and clear diffraction spots (inset in Fig. 3B), indicating the high crystallinity of the graphene sheets whilst very few graphene sheets (less than 1 percent) exhibited two typical sets of hexagonal diffraction pattern as shown in Fig. S4-A (see Supplementary information), indicating the non-Bernal stacking. The exact Bernal stacking for the graphene sheet was further confirmed by analyzing the profile of the SAED patterns. The relative intensity ratio $\mathrm{I}\{1210\} / \mathrm{I}\{1100\}$ was measured to be 0.4 and 2.1 for single layered and bilayered graphene sheets (Figs. S4-B and S4-C, see Supplementary information), respectively, which agreed well with other reported results ${ }^{24}$. The SAED and TEM analyses revealed that the single-layered graphene sheet was very limited (only two out of sixty sample size) whereas few-layered graphene sheets with Bernal stacking were dominant in the graphene yields.

The thickness of the as-synthesized graphene sheet was determined using atomic force microscopy (AFM) as shown in Figs. 4A and S5 (see Supplementary information). Based on sample analysis, the thickness distribution of the graphene sheets is statistically shown in Fig. 4B. No single-layered graphene (less than $1 \mathrm{~nm}$ ) was identified by the AFM tests. This could be due to the low constituent of single-layered graphenes in the samples as confirmed by the TEM and SAED results. The as-synthesized graphene sheets consisted mainly of sheets with less than $3 \mathrm{~nm}$.

Raman spectroscopy is widely used to characterize the structural and electron properties of graphene ${ }^{25-27}$. Fig. 5A represents a typical Raman spectrum of the sample. Several peaks are clearly visible at 1330 (D-mode), 1575 (G-mode), 1615 ( $\mathrm{D}^{\prime}$-mode), 2660 (2D-mode), 2925 (D + G-mode) and $3220 \mathrm{~cm}^{-1}\left(2 \mathrm{D}^{\prime}\right.$-mode $)$ as shown in Fig. $5 \mathrm{~A}^{28}$. In particular, the $2 \mathrm{D}$ and $\mathrm{G}$ bands represent the key features of Raman spectroscopy for the identification of graphene sheets. The $2 \mathrm{D}$ band peak at $2660 \mathrm{~cm}^{-1}$ is due to the highest optical branch phonons near the $\mathrm{K}$ point at the Brillouin zone boundary and the

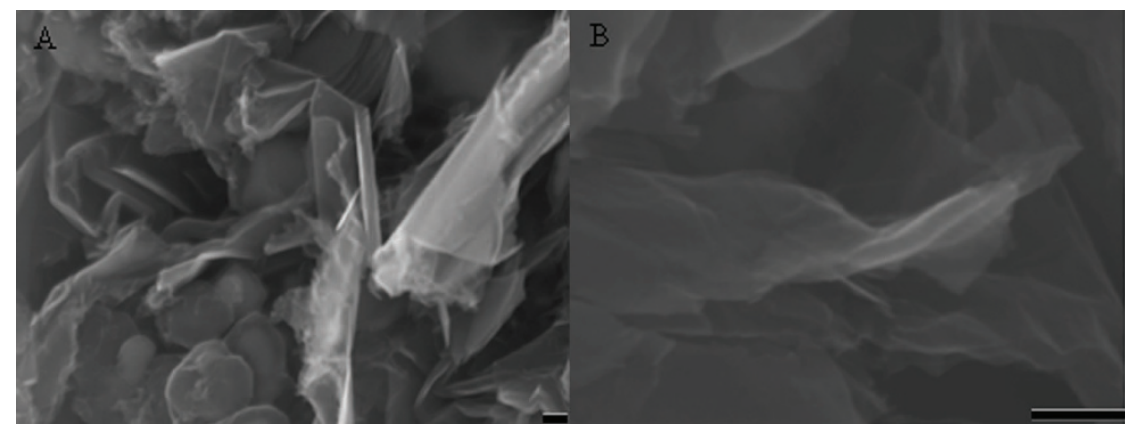

Figure $2 \mid$ SEM micrographs of the as-synthesized graphene sheets obtained at $1000^{\circ} \mathrm{C}$ under ambient pressure. (A) Low magnification, and (B) high magnification. The scale bars are $100 \mathrm{~nm}$. 

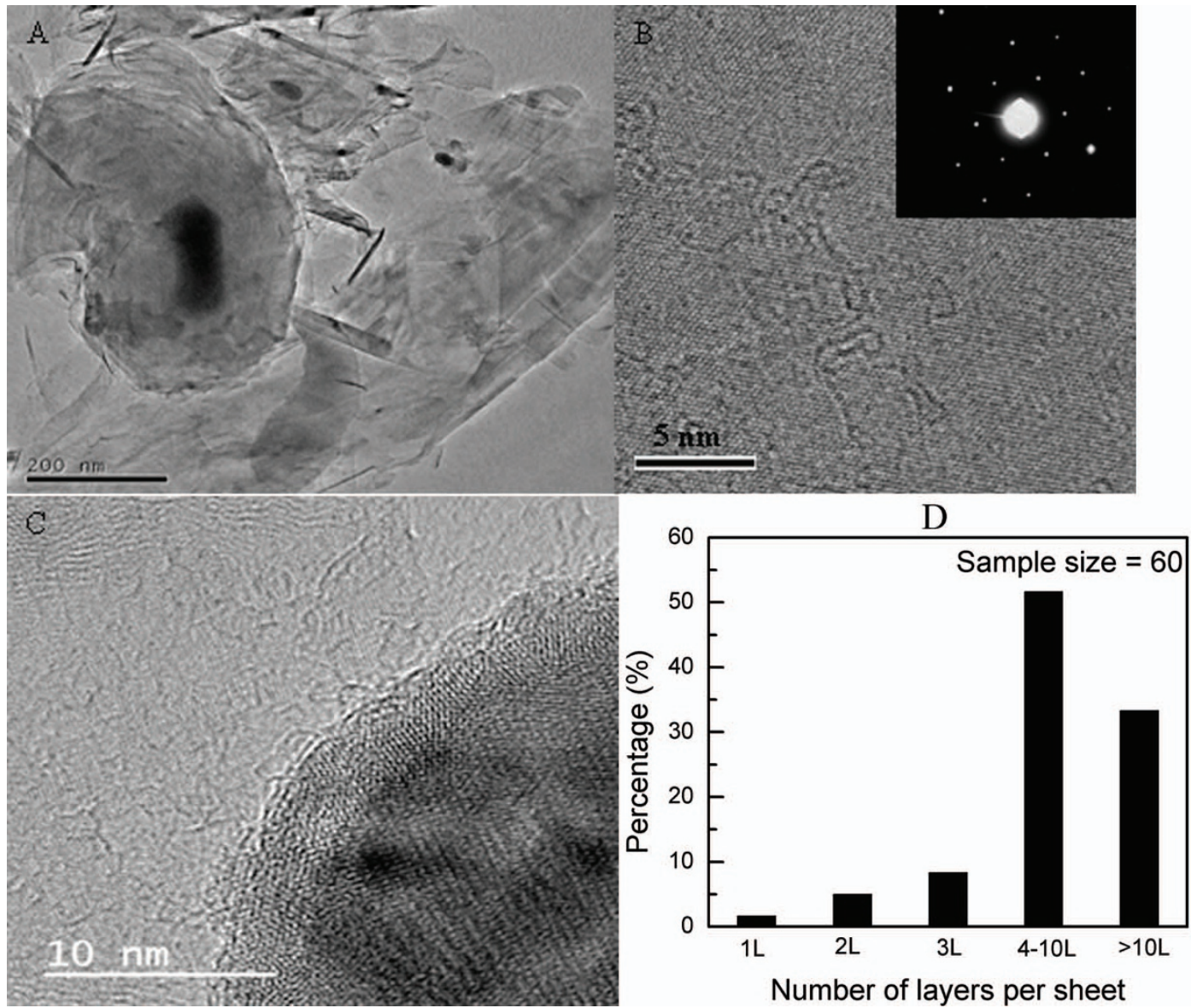

Figure $3 \mid$ TEM micrographs of graphene sheets obtained at $1000^{\circ} \mathrm{C}$ under ambient pressure. (A) As-synthesized graphene powder, (B) a typical graphene sheet (inset in (B): SAED pattern of the graphene sheet), (C) the interface between iron catalyst and graphene layers, and (D) the statistical distribution of the number of layers per graphene sheet.

$\mathrm{G}$ band peak at $1575 \mathrm{~cm}^{-1}$ is due to the two-fold degenerate $\mathrm{E}_{2 \mathrm{~g}}$ mode at the $\Gamma$-point. The relative intensity ratio of $\mathrm{I}_{2 \mathrm{D}} / \mathrm{I}_{\mathrm{G}}$ can be used to distinguish the number of layers of graphene sheets. The integrity intensity ratio $\mathrm{I}_{2 \mathrm{D}} / \mathrm{I}_{\mathrm{G}}$ of $>2,1-2$, and $<1$ correspond to singlelayered, double-layered and many-layered graphene, respectively ${ }^{19,29}$. For a good comparison, the spectra were normalized to give approximately the same relative intensity for all the samples. Fig. S6 (see Supplementary information) shows the spectra of graphene sheets with different layers. In Fig. $S 6 \mathrm{~A}$, when the $\mathrm{I}_{2 \mathrm{D}} / \mathrm{I}_{\mathrm{G}}$ intensity ratio is 1.6 and the full width at half maximum (FWHM) of 2D band is $63 \mathrm{~cm}^{-1}$, then this $I_{2 D} / I_{G}$ ratio verified a bilayered graphene sheet. With increasing number of layers, the intensity ratio of $\mathrm{I}_{2 \mathrm{D}} / \mathrm{I}_{\mathrm{G}}$ decreased whilst the FWHMs of the 2D peaks increased (see Fig. S6B, see Supplementary information). No single-layered graphene sheet was identified by the Raman test. The D band peak at $1330 \mathrm{~cm}^{-1}$ is a disorder-activated mode. The intensity of $\mathrm{D}$ band peak decreased
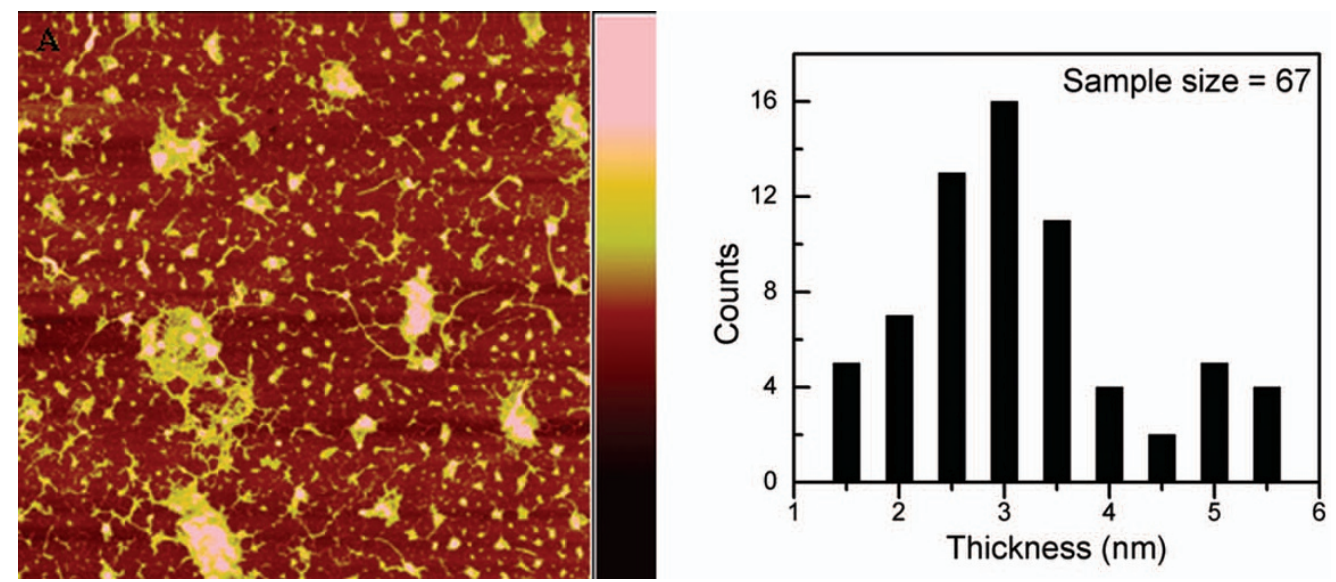

Figure $4 \mid$ AFM micrograph of graphene sheets obtained at $1000^{\circ} \mathrm{C}$ under ambient pressure. (A) A typical AFM micrograph of the as-synthesized graphene sheets after being transferred to a $\mathrm{SiO}_{2} / \mathrm{Si}$ substrate. The scanning area is $10 \times 10 \mu \mathrm{m}$ and the colour bar (right) is $20 \mathrm{~nm}$, and (B) the histogram of the thickness of the graphene sheets. 

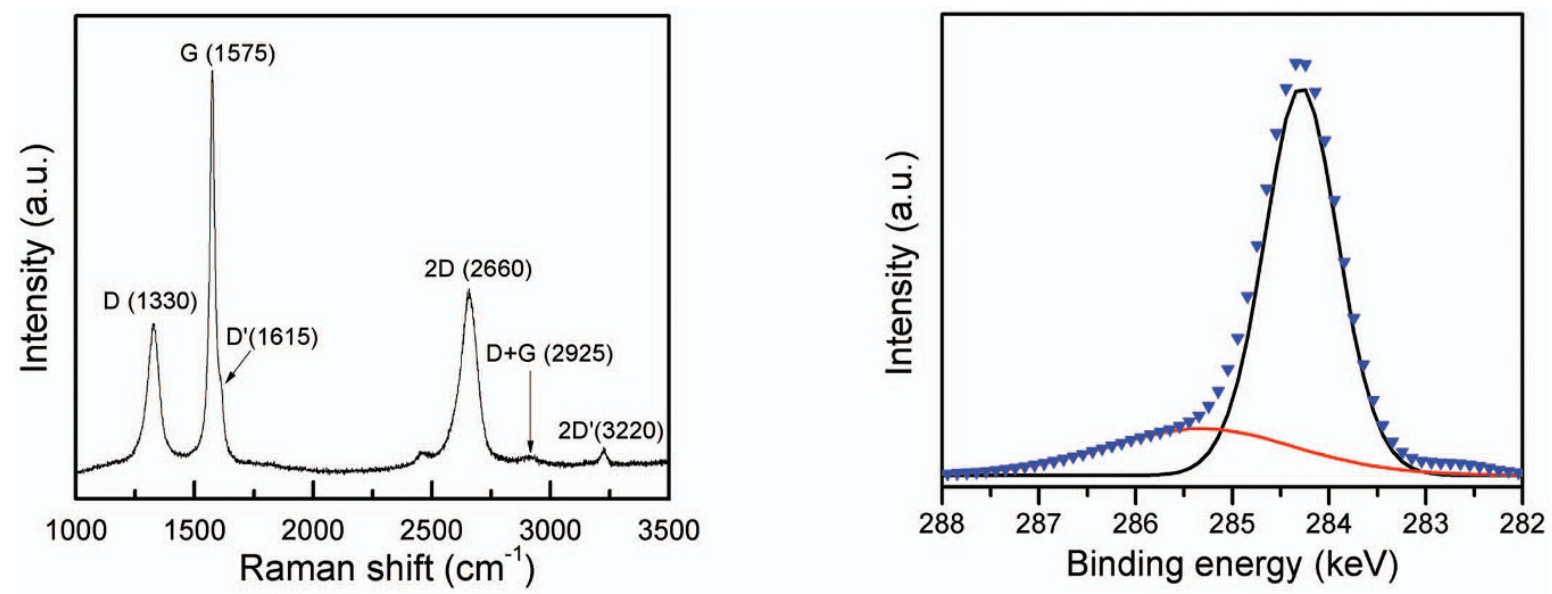

Figure $5 \mid$ Raman and XPS spectra of as-synthesized graphene sheets obtained at $1000^{\circ} \mathrm{C}$ under ambient pressure. (A) A typical Raman spectrum of the graphene sheet, and (B) C 1s core level XPS spectrum.

with increasing number of layers (see Fig. S6C, Supplementary information). It is believed that the intensity of $\mathrm{D}$ peak is closely related to the defects, i.e., edges, ripples and folders, in graphene sheets. The relatively high intensity of $\mathrm{D}$ band peaks in the samples in this study as compared with another study ${ }^{5}$ could possibly be due to the overlapping folders and edges which were captured by the laser spot during the Raman test.

The phase impurities of the as-synthesized graphene sheets, the graphene sheets after acid etching and the graphene sheets after sedimentation separation were examined by X-ray diffraction. Several distinctive peaks were well indexed to hexagonal carbon such as (002) and (101) planes, are constitutive of few-layered and multilayered graphene sheets, and body-centred cubic (bcc) iron crystal (Fig. S7, see Supplementary information). No iron carbide phase was observed in the XRD pattern. To further investigate the composition of the as-synthesized graphene sheets, sedimentation separations using a centrifuge were carried out. The results indicated that the as-synthesized product consisted of $48 \mathrm{wt} . \%$ of few-layered graphene sheets (supernatant) and $52 \mathrm{wt} . \%$ of multi-layered graphene sheets and catalyst particles (sediment). XRD patterns and TEM micrographs revealed that the supernatant consisted mainly of few-layered graphene sheets and the sediment consisted of catalysts wrapped by multi-layered graphene sheets. Energy dispersive X-ray (EDX) and $\mathrm{X}$-ray photoelectron spectroscopy (XPS) were used to analyze the elemental composition of the as-synthesized graphene sheets. The as-synthesized product consisted of carbon and iron only as shown in Fig. S8 (see Supplementary information). The C 1s core level XPS spectrum is shown in Fig. 5B. Two components in the spectrum at approximately 284.4 and $285.2 \mathrm{keV}$ were identified as the $\mathrm{sp}^{2}$ bonded carbon atoms and the $\mathrm{sp}^{3}$-bonded carbon atoms, respectively. These $\mathrm{sp}^{3}$-bonded carbon atoms were mainly present at the periphery of the graphene sheets and the defective dangling bonds. The intensity of $\mathrm{sp}^{2}$ carbon atoms was significantly dominant over that of $\mathrm{sp}^{3}$ carbon atoms as shown in Fig. 5B, indicating the high quality of the resulting graphene sheets.

To measure the bulk electrical conductivity of the graphene sheets, $0.3 \mathrm{~g}$ of the as-synthesized graphene powder was pressed into a disc of $12 \mathrm{~mm}$ and a bulk density of $1.6 \mathrm{~g} / \mathrm{cm}^{3}$ under a hydraulic force. The electrical conductivity was measured by a four-point probe method using a probe meter (Prob4) at room temperature. Eight measurements were carried out on the sample and the average value was reported. The thickness of the disc was measured using a digital micrometer. The bulk electrical conductivity of the graphene sheet powder was measured to be $59.5 \pm 3.0 \times 10^{2} \mathrm{~S} \mathrm{~m}^{-1}$, which was much higher than the reported value of $0.05 \mathrm{~S} \mathrm{~m}^{-130}$.

\section{Discussion}

In contrast to the CVD methods to produce graphene sheets as reported in the published literature, the method reported in this study is a continuous process, which does not involve a cooling precipitation process during the production of graphene sheets. The growth of graphene sheets by the thermocatalytic decomposition of methane could occur at temperatures ranging from 850 to $1050^{\circ} \mathrm{C}$. At a lower temperature, i.e., $850^{\circ} \mathrm{C}$, some carbon nanofibres were observed in the final product while at a high temperature of $1100^{\circ} \mathrm{C}$, self-decomposition of methane occurred, resulting in some carbon nanospheres (Figs. S9 and S10, see Supplementary information). The reaction temperature also played an important role on the growth kinetics of graphene sheets. Fig. 6A shows the graphene yield rate as a function of time at different temperatures. The initial graphene yield rates at the reaction temperatures of 850,950 and $1000^{\circ} \mathrm{C}$ were $6.1,12.4$ and $19.6 \mathrm{mg} / \mathrm{min}$ per $\mathrm{g}$ of catalyst, respectively. With increasing reaction time, the growth yield rate of graphene sheets showed distinct characteristics at different reaction temperatures. The graphene yield rate monotonically decreased with increasing reaction time at $1000^{\circ} \mathrm{C}$ whereas the yield rate increased with increasing reaction time at $850^{\circ} \mathrm{C}$. At a temperature of $950^{\circ} \mathrm{C}$, the graphene yield rate increased initially and then decreased thereafter. The decrease in isothermal yield rate of graphene sheet growth with increasing reaction time at high reaction temperatures could be due to the increased coverage of graphene layers over the surfaces of the iron particles, thereby preventing the methane from reaching the iron catalyst surface. The dependence of the yield rate of graphene sheet on the reaction temperature is closely related to the continuous graphene growth mechanism on the iron particles. The graphene growth mechanism depends on the dissociation rate of the methane on the catalyst particle surfaces and the diffusion rate of the graphene through the catalyst particles. If these two rates are balanced, graphene growth will persist. If the dissociation rate is faster than the diffusion rate, graphene sheets will slowly accumulate on the catalyst surface and eventually graphene sheets cease to grow when the catalyst surfaces are completely covered by graphene sheets ${ }^{31}$. At a high reaction temperature of $1000^{\circ} \mathrm{C}$ in Fig. $6 \mathrm{~A}$, the initial yield rate of graphene sheets was high because the high temperature brought forth a high dissociation rate of methane, resulting in a high yield rate of graphene sheets at the beginning but thereafter the yield rate continued to decrease due to the coverage of graphene sheets over the iron catalyst particles. The reverse was true for the lower reaction temperature of $850^{\circ} \mathrm{C}$ where the yield rate of graphene sheets continued to increase. By integrating the yield rate curves in Fig. 6A, the overall graphene sheet yields as a function of time were obtained as 

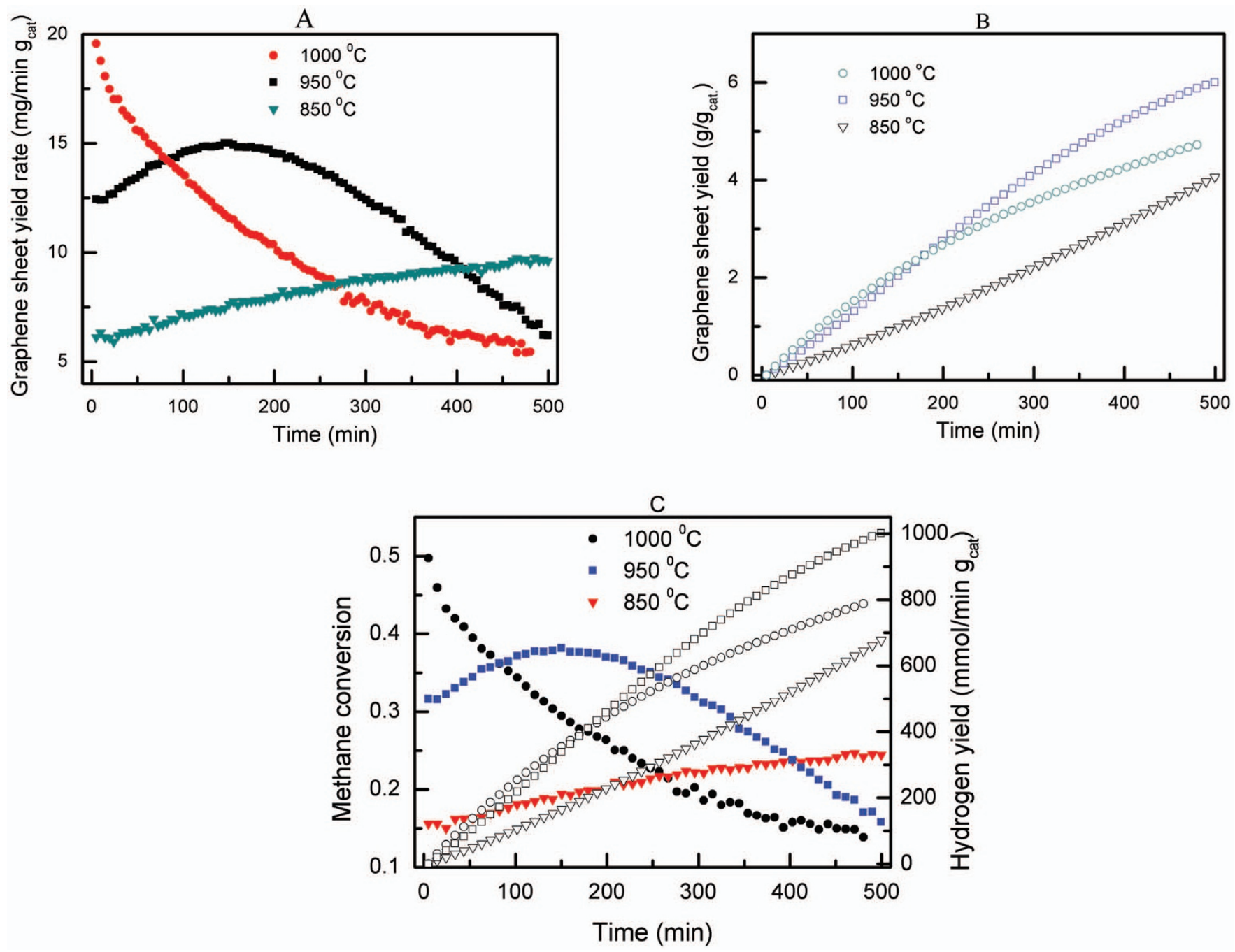

Figure 6 Graphene sheet production yield rate and yield from methane decomposition at 850,950 and $1000^{\circ} \mathrm{C}$ under ambient pressure.

Production of hydrogen as a by-product from methane decomposition. (A) Graphene yield rate, and (B) graphene yield as a function of reaction time.

(C) Methane conversion and hydrogen yield as a function of reaction time.

shown in Fig. 6B. The graphene yields were 4.1, 6.0 and $4.7 \mathrm{~g}$ per $\mathrm{g}$ of catalyst at reaction temperatures of 850,950 and $1000^{\circ} \mathrm{C}$, respectively, at the end of the tests. It should be noted that the graphene yield could still be further increased by increasing the reaction time and optimizing the feedstock composition. To reinforce the high graphene sheet yield rate (Fig. 6A) and the large-scale synthesis of graphene sheet (Fig. 6B), the corresponding methane conversion and the hydrogen yield as a function of reaction time are shown in Fig. 6C. The significant methane conversion verified the high graphene yield rate whilst the high hydrogen yield further confirmed the large-scale synthesis of graphene sheet. The additional important aspect of the thermocatalytic decomposition of methane is the generation of hydrogen as a by-product which certainly increases the economic benefits of this process. Due to the relative active properties of the iron particles, the as-synthesized graphene sheets could be easily purified using $\mathrm{HCl}$ ( $5 \mathrm{~mol} \%$ concentration) to dissolve the catalyst particles without significant changes to the morphology of the graphene sheets (Fig. S11, see Supplementary information).

This study demonstrated that, similar to carbon nanotubes, graphene sheets could be continuously synthesized under isothermal catalytic decomposition of methane without a cooling precipitation process. The present method exhibits an extremely high yield and efficiency, producing $6 \mathrm{~g}$ of graphene sheets per $\mathrm{g}$ of catalyst, which is 60 times the current yield reported ${ }^{21}$. This method could be easily scaled up and has the greatest near-term potential for industrial production of graphene sheets. The feasibility of large-scale production of graphene sheets could further pave the ways for its wide applications in devices such as high frequency transistors, batteries, sensors and solar cells.

\section{Methods}

Although iron has been widely used as a catalyst for the production of carbon nanotubes and carbon nanofibres, very few articles ${ }^{32,33}$ had reported on the use of iron as a catalyst for the production of graphene. In this study, iron oxide nanorods were used as the catalyst precursors and were reduced in-situ to obtain iron particles for the synthesis of graphene sheets by the thermocatalytic decomposition of methane. The iron oxide nanorods were synthesized by a simple precipitation method (see Supplementary information, Synthesis of iron oxides).

In a typical process of graphene sheet synthesis, $0.2 \mathrm{~g}$ of iron oxide crystals was loaded into a quartz glass tube and in-situ reduced to iron catalysts in an electric furnace using hydrogen. After the complete reduction of the iron oxides to iron particles, a mixture of methane and nitrogen with a volume ratio of methane to nitrogen of $5: 20$ was introduced at a flowrate of $25 \mathrm{ml} / \mathrm{min}$ with methane as the carbon source. The thermocatalytic decomposition of methane occurred and graphene sheets were continuously produced at various reaction temperatures of 850 , $900,950,1000$ and $1050^{\circ} \mathrm{C}$ under ambient pressure.

1. Novoselov, K. S. et al. Electric field effect in atomically thin carbon films. Science 306, 666-669 (2004).

2. Hernandez, Y. et al. High-yield production of graphene by liquid-phase exfoliation of graphite. Nature Nanotech. 3, 563-568 (2008). 
3. Eda, G., Fanchini, G. \& Chhowalla, M. Large-area ultrathin films of reduced graphene oxide as a transparent and flexible electronic material. Nature Nanotech. 3, 270-274 (2008)

4. Li, D., Muller, M. B., Gilje, S., Kaner, R. B. \& Wallace, G. G. Processable aqueous dispersions of graphene nanosheets. Nature Nanotech. 3, 101-105 (2008).

5. Kim, K. S. et al. Large-scale pattern growth of graphene films for stretchable transparent electrodes. Nature 457, 706-710 (2009).

6. Reina, A. et al. Large area, few-layer graphene films on arbitrary substrate by chemical vapour deposition. Nano Lett. 9, 30-35 (2009).

7. Gong, Y. P. et al. Layer-controlled and wafer-scale synthesis of uniform and highquality graphene films on a polycrystalline nickel catalyst. Adv. Funct. Mater. 22, 3151-3159 (2012).

8. Kwon, S. Y. et al. Growth of semiconducting graphene on palladium. Nano Lett. 9 , 3985-3990 (2009).

9. Sutter, P., Hybertsen, M. S., Sadowski, J. T. \& Sutter, E. Electronic structure of fewlayer epitaxial graphene on $\mathrm{Ru}(0001)$. Nano Lett. 9, 2654-2660 (2009).

10. Sutter, P. W., Flege, J. I. \& Sutter, E. A. Epitaxial graphene on ruthenium. Nature Mater. 7, 406-411 (2008).

11. Coraux, J., N'Diaye, A. T., Busse, C. \& Michely, T. Structural coherency of graphene on $\operatorname{Ir}(111)$. Nano Lett. 8, 565-570 (2008).

12. Li, X. S. et al. Large-area synthesis of high-quality and uniform graphene films on copper foils. Science 324, 1312-1314 (2009).

13. Robertson, A. W. \& Warner, J. H. Hexagonal single crystal domains of few-layer graphene on copper foils. Nano Lett. 11, 1182-1189 (2011).

14. Gao, L., Guest, J. R. \& Guisinger, N. P. Epitaxial graphene on $\mathrm{Cu}(111)$. Nano Lett. 10, 3512-3516 (2010).

15. Wang, H. et al. Controllable synthesis of submillimeter single-crystal monolayer graphene domains on copper foils by suppressing nucleation. J. Am. Chem. Soc. 134, 3627-3630 (2012)

16. Zenasni, A., Delamoreanu, A. \& Rabot, C. Free-suspended graphene synthesis via carbon diffusion through platinum-based metal. Appl. Phys. Lett. 100, 151907 (2012)

17. Peng, M. Z. et al. High-resolution in situ and ex situ TEM studies on graphene formation and growth on Pt nanoparticles. J. Catal. 286, 22-29 (2012).

18. Chen, J. Y. et al. Oxygen-aided synthesis of polycrystalline graphene on silicon dioxide substrates. J Am. Chem. Soc. 133, 17548-17551 (2011).

19. Medina, H. et al. Metal-free growth of nanographene on silicon oxides for transparent conducting applications. Adv. Funct. Mater. 22, 2123-2128 (2012).

20. Emtsev, K. V. et al. Towards wafer-size graphene layers by atmospheric pressure graphitization of silicon carbide. Nature Mater. 8, 203-207 (2009).

21. Wang, X. B. et al. Large-scale synthesis of few-layered graphene using CVD. Chem. Vap. Deposition. 15, 53-56 (2009).

22. Zhu, Y. W. et al. Graphene and graphene oxide: synthesis, properties, and applications. Adv. Mater. 22, 3906-3924 (2010)
23. Meyer, J. C. et al. The structure of suspended graphene sheets. Nature 446, 60-63 (2007).

24. Dato, A. et al. Substrate-free gas-phase synthesis of graphene sheets. Nano Lett. 8 , 2012-2016 (2008).

25. Graf, D. et al. Spatially resolved raman spectroscopy of single- and few-layer graphene. Nano Lett. 7, 238-242 (2007).

26. Ferrari, A. C. et al. Raman spectrum of graphene and graphene layers. Phys. Rev. Lett. 97, 187401 (2006).

27. Gupta, A. et al. Raman scattering from high-frequency phonons in supported ngraphene layer films. Nano Lett. 6, 2667-2673 (2006).

28. Rao, C. N., Sood, A. K., Subrahmanyam, K. S. \& Govindaraj, A. Graphene: the new two-dimensional nanomaterial. Angew. Chem. Int. Ed. 48, 7752-7777 (2009).

29. Shan, C. S. et al. Facile synthesis of a large quantity of graphene by chemical vapour deposition: an advanced catalyst carrier. Adv. Mater. 24, 2491-2495 (2012).

30. Choucair, M., Thordarson, P. \& Stride, J. A. Gram-scale production of graphene based on solvothermal synthesis and sonication. Nature Nanotech. 4, 30-33 (2009).

31. Lua, A. C. \& Wang, H. Y. Decomposition of methane over unsupported porous nickel and alloy catalyst. Applied Catalysis B: Environmental. 132-133, 469-478 (2013).

32. An, H., Lee, W. J. \& Jung, J. W. Graphene synthesis on Fe foil using thermal CVD. Curr. Appl. Phys. 11, 81-85 (2011).

33. Xue, Y. Z. et al. Synthesis of large-area, few-layer graphene on iron foil by chemical vapour deposition. Nano Res. 4, 1208-1214 (2011)

\section{Author contributions}

Y.S. and A.C.L. wrote the main manuscript text and Y.S. prepared figures 1-6. All authors reviewed the manuscript.

\section{Additional information}

Supplementary information accompanies this paper at http://www.nature.com/ scientificreports

Competing financial interests: The authors declare no competing financial interests.

How to cite this article: Shen, Y. \& Lua, A.C. A facile method for the large-scale continuous synthesis of graphene sheets using a novel catalyst. Sci. Rep. 3, 3037; DOI:10.1038/ srep03037 (2013).

This work is licensed under a Creative Commons AttributionBY NG ND NonCommercial-NoDerivs 3.0 Unported license. To view a copy of this license visit http://creativecommons.org/licenses/by-nc-nd/3.0 